\title{
Neural \& Predictive Effects of Verb Argument Structure
}

By

\author{
Daniel Di Giovanni
}

\begin{abstract}
Thesis Submitted to the Faculty of Graduate and Postdoctoral Affairs in partial fulfillment of the requirements for the degree of
\end{abstract}

Master of Cognitive Science

in Cognitive Science

Carleton University

Ottawa, Ontario

(C)2016

Daniel DiGiovanni 


\begin{abstract}
This project used several brain imaging techniques to examine the mechanisms of verbs. Specifically, it compared material central to the meaning of the verb (linguistic arguments) to material peripheral to the meaning of the verb (linguistic adjuncts). This studies provides electroencephalogram (EEG) recordings of event-related potentials (ERPs), event-related spectral perturbations (ERSP), as well as other electro-physical analyses that not only suggest a distinction exists but that different underlying neurological mechanisms process them. Participants were exposed (native monolingual English speakers) to audio English sentences, among these were non-sense sentences that only differed by whether the final word was an argument or an adjunct. I then compared these to well-formed sentences. The study found that the adjunct condition elicited distinct potentials and reactions from other conditions. The ERP results showed that non-sense arguments generated an early left anterior negativity (ELAN), which is assumed to represent a local syntactic error and a N400, assumed as a semantic error. Noticeably the non-sense adjunct condition only elicited a P600 signal, late error processing. The well-formed sentence demonstrated no significant signals. All of this strongly suggests a distinction between the conditions. Further investigation suggests that different mechanisms underlying these signals.
\end{abstract}




\section{Acknowledgments}

I wish to express my sincere gratitude to all the people that made the research presented in this thesis possible. I am very grateful to the members of my thesis committee, Ida Toivonen, Georg Northoff, and Daniel Siddiqi. Ida Toivonen has taught me a great deal about what it takes to be a successful academic over the course of this project, and has been an inspiration to me. Without her guidance and constant support this project would have never happened. Her expertise on language has been invaluable over the course of this entire thesis. I am extremely grateful for all of Ida's guidance and advice. I am also indebted to Georg Northoff for providing me access to his EEG lab, and for teaching and advising me on experimental methods and techniques, as well as for opening the world of neuroscience to me. His consistent support has been indispensable. Daniel Siddiqi has also been a constant source of guidance and inspiration during the course of this thesis. He has been a great mentor to me and has taught me many things about language, and about being a teacher. I cannot thank him enough for his support. Each committee member had a great influence not only on this thesis, but also in making this project a satisfying, constructive, and enjoyable one. I would also like to extend an additional thanks to Annemarie Wolff for teaching me the details of EEG work and analysis. Finally I would like to thank my parents, who have always encouraged and supported me. This thesis is dedicated to them. 


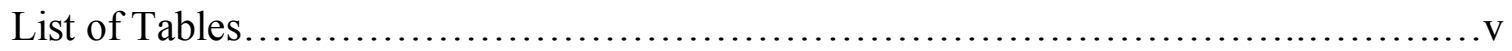

List of Figures..........................................................

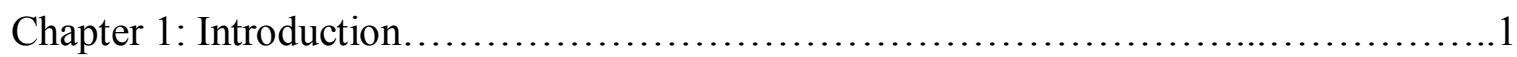

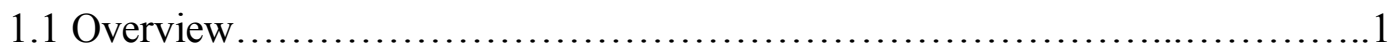

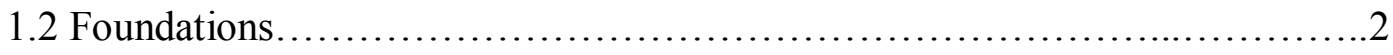

Chapter 2: Arguments and Adjuncts............................................6

2.1 Defining Verb Argument Structure.....................................6

2.2 Issues Distinguishing Arguments from Adjunct................................9

2.3 Where is Argument Structure?........................................................... 11

Chapter 3: Electrophysiological Methods and Prediction............................ 15

3.1 Psychological Correlates of Alpha-band Activity.............................15

3.2 Internally and Externally Motivated Stimuli............................ 16

3.3 Treating Argument Structure as Externally Motivated......................19

3.4 Using Grooming Verbs as Effective Stimuli.............................21

Chapter 4: Methods...........................................................24

4.1 Overview and Design............................................. 24

4.1.1 Participants..................................................24

4.1.2 Experimental Design.....................................24

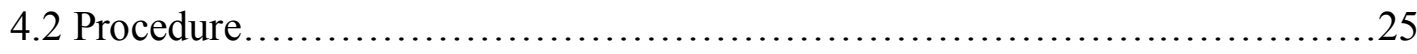

4.2.1 Electrophysiological recording ..............................25

4.2.2 Artifact Rejection........................................26

4.2.3 Event Related Potential Analyses..............................26 
4.2.4 Spectral Analyses...........................................26

4.2.5 Event Related Spectral Perturbation Analyses......................27

4.2.6 Inter Trial Coherence Analyses................................27

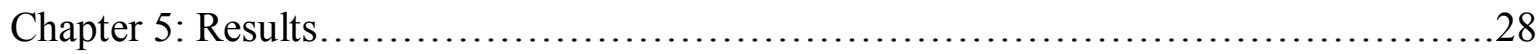

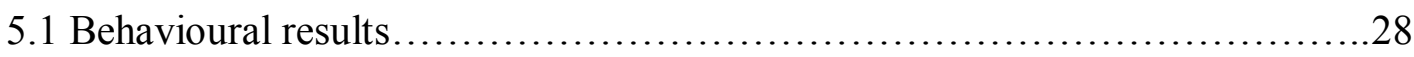

5.1.1 Descriptive statistics.........................................28

5.1.2 Effect of Sentence Types..........................................28

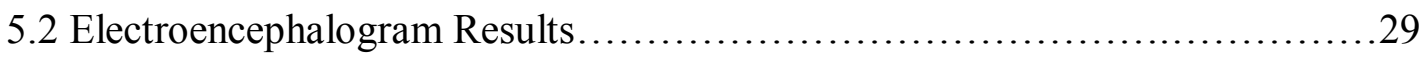

5.2.1 Event Related Potential Results................................29

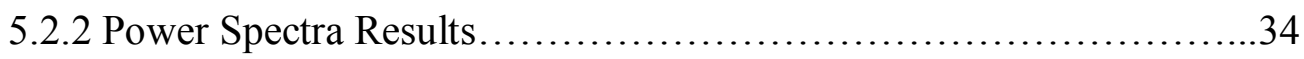

5.2.3 Time-Frequency Results........................................ 35

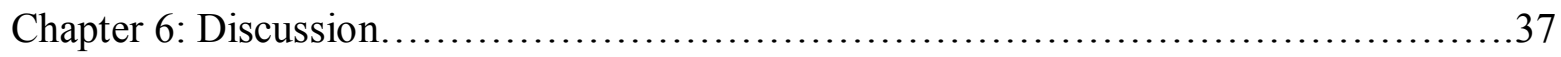

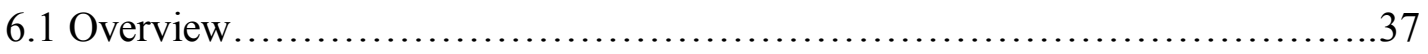

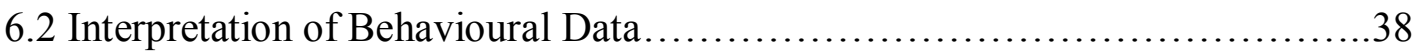

6.3 Interpretation of Event Related Potential Data................................39

6.4 Interpretation of the Power Spectra Data.....................................40

6. 5 Interpretation of Time-Frequency Data....................................41

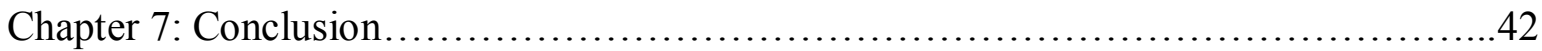

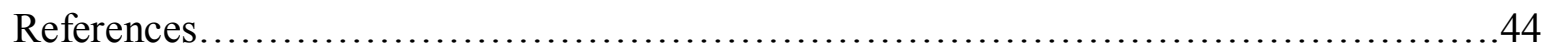

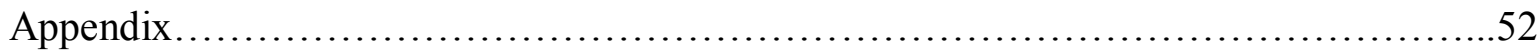




\section{List of Tables}

Table 1. Example of each condition defined by category and type. ............................ 24

Table 2. Means and Standard Deviations of the reaction time for each condition. .......... 28 


\section{List of Figures}

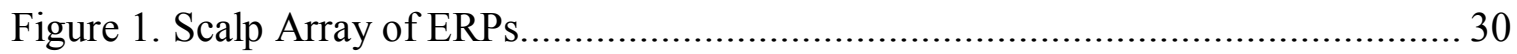

Figure 2. P600 and N400 ERP from channel CZ with degrees of error.

Figure 3. ELAN ERP from channel FC5, and degrees of error.

Figure 4. Power Spectral distribution from channel FCZ..................................... 34

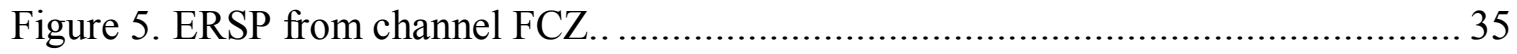

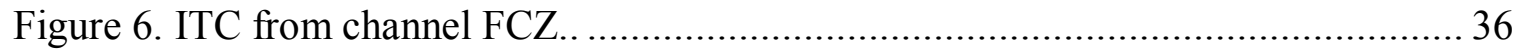




\section{Chapter 1: Linguistics and Neuroscience}

\subsection{Overview}

This project seeks to consider new ways in which neuroscience and language can inform each other. This is achieved by using language as a high-order cognitive function that can be manipulated to test newly developed neuroscience methodologies, namely quantitative electroencephalography (qEEG) methods of analysis. The hypothesis is that the predictive ability of online language processing is sensitive to the syntactic requirements of verbs and will have quantifiable neural effects. For example, phrases that modify the meaning of a verb phrase but are not a required syntactic component by the verb, such as adverbs, are impossible to predict by listeners, since there are no linguistic cues that necessitate their appearance. This is a different way of approaching the distinction between arguments and adjuncts. This project specifically examines internal arguments, otherwise known as verb complements and how they are distinct from adjuncts. This distinction will be explained more fully in chapter two. The idea though is that adverbs and other adjuncts must rely on more self-driven processing because they lack an external cue that can strictly dictate their purpose. On the other hand, the presence of arguments is determined by the verb, therefore the verb acts as an external cue for the argument. If adjuncts are more internally driven in processing then they should also correlate with neural signs of internal processing. Chapter three discusses the differences between externally driven decision making and internally driven decision making, and how arguments and adjunct fit into this, in more detail. Chapter three also elaborates on the neural correlates and mechanis ms for these phenomena. Chapter four through six describe an experiment that tested the proposed hypothesis and its results. Finally Chapter seven concludes the discussion, and speculates how future work might approach this subject. 


\subsection{Foundations}

Interestingly some of the earliest work done in neuroscience, during the $19^{\text {th }}$ century, was tied to the study of language. This history is laid out by Philips and Sakai's (2005) book Language and the Brain. ${ }^{1}$ The first intersection of neuroscience and linguistics was known as aphasiology, and studied the effect that brain injuries had on linguistic processing. This field grew out of the pioneering work of Pierre Paul Broca, who was the first to link particular areas of the brain to language processing. As a field surgeon he conducted numerous autopsies on people who had speaking disorders. He soon drew a link between speaking disorders and an area in the left frontal lobe, specifically near the posterior inferior frontal gyrus. Today this particular area of the brain is commonly known as Broca's area and represented as area 44 and 45 on Brodmann's map of the brain. Broca's work was some of the very first empirical data to link a particular brain area to a particular function, and is seen as the first real breakthrough in the field of neuroscience. Later, Carl Wernicke built on the work of Broca. He proposed that different areas of the brain were specific to distinct linguistic tasks. He found that Broca's area was typically responsible for speech production, and discovered a distinct area that seemed to handle speech comprehension. This area was located in the posterior section of the superior temporal gyrus in the left hemisphere, and is now known today popularly as Wernicke's area, and described as area 22 on Brodmann's map of the brain. The work of both Broca and Wernicke was seminal in the field of neuroscience and their discoveries would later be important in linguistics and the developing field of cognitive science. The term neurolinguistics was then coined in the 1950s by Alexander Luria in his book Problems in Neurolinguistics, and popularized by Harry Whitaker who founded the journal Brain and Language in 1974. Given

\footnotetext{
${ }^{1}$ Philips and Sakai (2005) is the source for the overview in this section.
} 
this history one would expect that language and neuroscience have had a long and fruitful relationship with each other. However, the two fields outside of Aphasia studies went down separate paths, so to speak, with less interaction than one might expect during the second half of the $20^{\text {th }}$ century. Only within the last couple of decades as neuroimaging techniques have improved has neuroscience started to really interact with linguistics again. Since then several language related brain responses have been identified, and neuroscience has become a widely used tool for linguists with various modern brain imaging technologies and time-sensitive electrophysiological techniques. Yet the current intersection between linguistics and neuroscience is not a strong as it could be, and the problematic aspects of neurolinguistics need to be addressed.

As touched on by Chomsky in New Horizons in the Study of Language and Mind (2000) and expanded on by psychologist and neuroscientist David Poeppel in his 2005 paper, Defining the relation between linguistics and neuroscience, as well as several other authors (Lorenzo, 2003; Chomsky 2005; Marantz, 2005; Boeckx \& Grohmann, 2007; Sidtis, 2007; Fedorenko \& Kanwisher, 2009; Kinsella, 2009; Di Sciullo \& Boeckx, 2011) there is a sentiment that neurolinguistics, and the kindred field of biolinguistics, are not as productive as they could potentially be. Most of this work claims a conceptual change is necessary to allow for a unification of linguistics, biology, and neuroscience. Since the technology currently does not exist to correlate linguistic elements directly with the fundamental biological units associated with neuroscience, it makes a direct reduction between theoretical linguistics and neuroscience difficult. For example, one simply cannot directly match the concept of a morpheme with a cellular ensemble, at least with the current state of imaging technology. Because a direct reduction is not possible there is a threat of a Granularity Mismatch, as different levels of 
representations are used in linguistics and in neuroscience and this can lead to vague metaphors on how brain structures relate to linguistic components.

This is not to say that there have not been many discoveries, or that neurolinguistic research has not made any progress. In fact there are many robust event-related potential (ERP) effects that have been discovered. For example, the N400 component has been a steady finding in neurolinguistic research since the 1980 s, and seems to be related to some sort of semantic violation (Kutas \& Federmeier, 2011). The existence of such components shows that the brain processes semantics differently than it processes syntax (Friederici et al., 2000); however the risk is in linking such a component too strongly to a theoretical representation. An ERP is just a change in polarization in response to stimuli; it is not a neural mechanism in and of itself, just an indication of a change of brain states in response to stimuli. The focus should be on how the actual computational mechanisms of the brain may affect mental states - supplemented by ERP evidence but not explained with it. So there is also the problem of relying too much on solely ERPs in neurolinguistics, when there exist many other neuroscience tools that neurolinguists could use to their advantage. As an example, cross-frequency couplings, that is coupling effects between different frequency bands, have recently been a hot topic in neuroscience as a mechanism for quick long distant communication between brain areas (Canolty and Knight, 2010). This is an example of a computational process used in the brain. Perhaps coupling mechanisms are an important component in the neuronal encoding of language, or as a means for how other cognitive functions such as memory interact with language processing. To my knowledge, however, there have yet to be any studies which look at such effects in language, and it is an area which could warrant investigation. This project utilizes event related analyses as well as some quantitative electroencephalogram techniques to gain insight into how language 
processing is reflected in neural mechanisms. This will be achieved by testing basic syntactic concepts, namely arguments and adjuncts, and analysing the neuronal activity that these different conditions generate. The goal is to demonstrate novel ways in which neuroscience and linguistics can interact and start to address some of the issues mentioned above. 
Chapter 2: Arguments and Adjuncts

\subsection{Defining Verb Argument Structure}

The following is a basic overview of the argument structure of verbs, similar to what may be discussed in an undergraduate course on syntax, provided by Kroeger's Analyzing syntax: a lexical-functional approach (2004). The purpose of this section is just to explain in general terms how verb argument structure is defined for the purpose of this study.

Kroeger defines verbs as things that describe events and states, and the argument structure of a verb is a representation of things that the verb needs to make sense. A verb may require an agent. The term agent is used to describe the thing that does, or causes, a particular action or event. A verb may also require a patient, and typically the term patient is used to refer to the thing that is acted upon. Consider the following examples:
(a) run $<$ agent $>$
(b) melt $<$ patient $>$
(c) kick < agent, patient $>$

The three verbs in (1) describe different events that have different participant requirements for the event to take place. The verb run needs an agent, that is to say it needs someone to do the act of running. This is different from melt which requires something to be acted on, but not necessarily something to do the melting, as in the phrase the ice cream melted, as in this case the melting is completely intelligible even without describing what is causing the melting event. Both run and melt only need one participant, in the case of run a participant that performs the action, or the agent, and in the case of melt a participant that is acted on, the patient, but there are some verbs that need multiple participants to make sense. In (1c) the verb kick requires two 
participants, both an agent (a thing to do the kicking) and a patient (the thing kicked) in order for the kicking event to be completed. These different participant requirements of verbs are known as their argument structure. The argument structure of a verb is closely related to the meaning of the verb and tied to the event that the verb describes, but does not necessarily capture the entire meaning of the event or all possible aspects of the event. The argument structure is usually a representation of the things needed to make describing the event grammatical; they do not always capture all the information that could be expressed when describing an event. Considering the verb run again, one may describe a running event with a time, a location, a manner, or a purpose.

(a) I run.

(b) I run at night in the park quickly to get exercise.

(c) *run at night in the park quickly to get exercise.

The sentence (2a) is perfectly grammatical with only an agent expressed (here marked in bold), and $(2 \mathrm{~b})$ is grammatical with the agent expressed but also with a time of day, a location, a manner, and a purpose expressed in the same sentence, however none of these things (all underlined) are actually required since (2a) is totally grammatical without them being expressed. They are all things that occur with a running event, but they are not essential to the meaning of a running event. The agent is essential though, and without the agent, like in $(2 \mathrm{c})$, the sentence is no longer grammatical. So from this example we can conclude that the agent is a part of the argument structure, but the extra underlined information is not. Generally the information present that is not part of the argument structure is expressed with adjuncts. Adjuncts change the overall meaning of a phrase but are not as closely associated with the meaning of the verb and therefore 
their absence does not cause ungrammaticality. Besides arguments generally being obligatory, while adjuncts are not, there are other aspects that suggest a distinction between the two. Adjuncts can be repeated in clauses they appear in, while verb arguments must be unique.

(a) *John gave Sue a gift to Mary

(b) John ran yesterday, during the afternoon, at around $\underline{3 p . m}$.

The sentence in (3a) is clearly not grammatical. The give requires three arguments: an agent to give, a thing given, and a recipient of the gift. (3a) however has two recipients for the gift, showing that the argument of the recipient cannot be repeated. Sentence ( $3 b)$ though has three time adjuncts (all underlined), and still seems to be completely grammatical.

Another difference between arguments and adjuncts is that sometimes verbs have selectional restrictions on what type of arguments can be used, whereas adjuncts have no such restrictions. For example, the verb drink requires an agent to do the drinking and a patient that is drunk, but the thing drunk cannot just be anything it needs to be a liquid. If one refers to a location using the verb drink though, there are no restrictions imposed by the verb where that location can be. The sentence (4a) is strange because drink selects things that are liquid to be its patient, and bolded argument is not a liquid. The adjunct in (4b), which is underlined, is just as ridiculous as the idea of drinking cardboard, but the sentence is not ungrammatical because the location of the drinking event is opaque to the verb and it cannot restrict something it does not care about.

(a) ?I drank cardboard.

(b) I drank soda in the planet core. 
In summary, arguments can be distinguished from adjuncts, because arguments are obligatory where adjuncts are optional, arguments must be unique where adjuncts can be repeated, and arguments have selectional restrictions determined by the verb where adjuncts do not.

\subsection{Issues Distinguishing Arguments from Adjunct}

Although useful for outlining the general difference between arguments and adjuncts, the above examples paint an overly simple picture of the argument adjunct distinction. Beth Levin and Rappaport Hovav (2005) describe several complications in their book Argument Realization. For one, it seems some arguments are not actually obligatory, meaning they can alternate between being overtly expressed and just being implied. Consider eat again, both I eat and I eat

dinner are acceptable phrases, so one may assume that the patient here is optional and therefore an adjunct to the verb. This seems unlikely though, as going by our earlier characteristics of adjuncts, the patient in this case is not repeatable (*I eat dinner chicken), and has a selectional restriction on edible things (?I eat ideas). Both of these characteristics are typical for arguments. So the distinction between what constitutes an argument and what constitutes an adjunct has some gray area, at least in a syntactic sense. Semantically, it is clear that an eating event requires a thing to be eaten.

In order to try and tease out the distinction in less obvious cases, several tests have been proposed to make the distinction clearer. However, there are still unclear cases. For example prepositional phrases that express instruments seem to be conceptually necessary, and pass several tests that suggest they are arguments, but also fail others (Barbu, 2015). The tests manipulate the structure of a given sentence to see whether these unclear cases are arguments or adjuncts. Structurally arguments and adjunct attach at different places, a common view is that 
arguments are sisters to the verb and adjuncts are sisters to the verb phrase. The diagnostic tests take advantage of this fact to tease out where the unclear cases are in the syntactic hierarchy. Despite these tests there are still many cases that are difficult to categorize, in particular prepositional phrases have been notoriously difficult (Larson, 1988; Marantz, 1984; Schutze \& Gibson, 1999). Furthermore, Needham and Toivonen (2011) discuss several ways that the tests are limited in their explanatory ability. Also, there is no agreed upon set of tests used to determine this distinction and a wide variety of tests to choose from. This suggests the tests lack power (Boland 2005). In order to deal with this dilemma, some have claimed that such a distinction between arguments and adjuncts exists on a scale and cannot be clearly defined (Grimshaw, 1990; Forker, 2014).

Perhaps the problem needs to be approached differently, since intuitively and empirically there seems to be a distinction. The intuition can be best described as follows:

"Adjuncts are always optional, whereas complements are frequently obligatory. The difference between them is that a complement is a phrase which is selected by the head, and therefore has an especially close relationship with the head; adjuncts, on the other hand, are more like 'bolt-on' extra pieces of information and don't have a particularly close relationship with the head." (Tallerman, 2005, 98)

The above definition provided by Tallerman seems to tap into the intuitive differences between arguments and adjuncts. Needham and Toivonen (2011) proposed a way to explain these inbetween cases, while maintaining the intuitive difference between arguments and adjuncts. They claim that the in-between cases are examples of derived arguments. Simply put, these in-between cases are derived from rules that can add arguments. For example, their rule for dealing with the problematic instrument case mentioned above is to suggest that they are the result of the rule:

\section{Optionally add an oblique instrument argument to verbs whose first argument is}

an agent. 
This allows the instrument to exist as an argument at the level of expression, but is not an inherent part of the verb, so it is not actually a part of the verb's argument structure. This could account for these in-between cases. Moreover, there are good reasons to believe that the distinction exists for empirical reasons, as data collected from lay people have been shown to intuitively display a distinction (Barbu, 2015). There are also many other empirical studies that have demonstrated that a distinction exists (Shapiro et al., 1989; Britt, 1994; Schütze \& Gibson, 1999; Boland and Blodgett, 2006; Tutunjian and Boland, 2008; Lee and Thompson, 2011). In sum, the distinction between arguments and adjuncts is not easy to pinpoint and there are some unclear cases. However, the distinction is still widely assumed in the linguistic literature, and there are strong reasons to believe that there is something to it. A goal of this project is to add to the empirical evidence for a distinction between arguments and adjuncts.

\subsection{Where is Argument Structure?}

There are some neurolinguistic studies that show argument structure is captured by neural activity. Thompson et al. (2007) suggests that neuron activation is proportional to the structural complexity of the verb. Complexity here refers to the number of syntactic arguments a verb takes, with more arguments involving a more complex structure. They conducted a functional magnetic resonance imaging (fMRI) study, where participants were exposed to single word stimuli. The point was to associate differences in argument structure to difference in activation patterns. They found significant differences in activation associated with verbs with one argument and verbs with two and three arguments. They found that neural activity in the inferior parietal region increases as argument complexity increases. Specifically they found that more complex verbs (ditransitive and transitive) used far more neural processing than simpler verbs 
(intransitive). In particular it seemed the angular gyrus (BA39) was active in the processing of more complex verb structures. This is line with agrammatic aphasic patients, who struggle with processing more with complex verbs when there is damage to these regions. They claim this finding makes sense with existing theoretical linguistics theories, and conclude by saying that most linguists would accept ditransitive and transitive verbs as having more complex structures, and they claim this finding suggests this is motivated by the syntactic properties that are inherent in verbs, namely their argument structures.

A 2008 fMRI study by den Ouden et al. made similar findings for verb speech production as Thompson et al. (2007) did for listening comprehension. They presented participants with visual stimuli. The stimuli were pictures and videos of actions being carried out. The participants were asked to name the actions. When the participants did this, they found increased activation during transitive verb naming, over intransitive naming. Again, this suggests that an increase in structural complexity leads to an increase in neural activation. This is evidence that argument structure is a real phenomenon and that it is relevant in neuronal computation. This neural activity occurred in all of the typically identified language areas, such as Broca's Area (BA 44, 45), and the supramarginal and angular gyri, with a left hemisphere domination. They also saw neural recruitment in the anterior regions of the peri-sylvian language network, and the ventral and visual pathways that did not occur in the 2007 study. This is likely caused by the pictographic stimuli used and the nature of speech production versus listening processing. They claim that extra activation is consistent with previous work that attributes those areas with object recognition and representation.

A more recent study by Meltzer-Asscher et al. (2012) built on the previous studies mentioned and claimed that the more neural recruitment used by complex verb structures is 
evidence of the lexicalization of specific argument structures in verbs. Lexicalization refers to the features that are associated with a specific word. According to this view, for example, a ditransitive verb has three inherent roles that need to be fulfilled, and these required roles determine what kind of noun phrases the verb needs to be grammatical. Meltzer-Asscher's study sought to demonstrate that these roles are inherent to the verb by comparing neuronal activity associated with intransitive verbs and verbs with alternating transitivity. The study found that when alternating transitivity verbs took on an intransitive frame they resulted in more activation of the angular and supramarginal gyri bilaterally than non-alternating intransitives. Their conclusion was that the full structure is inherent to the verb whether it is fully expressed or not, since the alternating verbs require more processing, suggesting they have more lexical information than non-alternating verbs. This lends support to the hypothesis that this information is lexicalized (a component of the lexeme, or word itself).

Besides fMRI data there is also psycholinguistic data that suggest that argument structure is an inherent property of verbs, in other words that argument structure is lexicalized and therefore strictly determined by the verb used. Julie Boland (2005) presents an eye-tracking study. In this study participants listen to various recordings of sentences while their eye movement was tracked. Also while listening to these recordings several images were displayed in front of them. She reports that participants tended to look at the pictures that were relevant to the verb even while the verb was still playing, meaning these objects were associated with the typical theta roles of the verb. This is congruent with findings by Altmann and Kamide (1999), which found faster eye movements to things that fulfill a verb's theta role. Boland goes on to say that in the case of where it is not clear what the argument structure is, participants preferred the more frequent structure, i.e. ...suggest an idea (no recipient) is preferable to ...suggest an idea to 
John (with a recipient), because the recipient is often not expressed. Even though a case can be made that a suggesting event always conceptually needs a recipient, she claims that this is not lexicalized to the verb as it is not frequent enough to warrant lexicalization. She concludes that verb structures are recognized through the verb itself and then are weighted by their frequency.

Julie Boland's work on frequency effects and argument structure suggests that arguments are in fact lexicalized. This would mean that adjuncts are not lexicalized and therefore parsed differently. Interestingly the finding that people tend to predict subsequent arguments based on the expressed verb lends support to the proposed hypothesis that lexicalized verb features inform the listener's prediction of upcoming words in a phrase. All of this suggests that any decision making in predicting upcoming words is motivated by the processing of the verbs themselves, and therefore any upcoming adjuncts are not predictable in this manner. Once again, this is referring to the internal argument, or complement, not the external argument, since it is the internal argument that appears after the verb, and is tied closer to the verb. Hence, in subsequent sections when the term argument is used, it is referring to the internal argument. 
Chapter 3: Electrophysiological Methods and Prediction

\subsection{Psychological Correlates of Alpha-band Activity}

The oscillations of brain activity are grouped into several bands of different frequency ranges. The delta band is defined as oscillations with a frequency of $1 \mathrm{~Hz}$ to $4 \mathrm{~Hz}$, the theta band is defined as $5 \mathrm{~Hz}$ to $7 \mathrm{~Hz}$, the alpha band is $8 \mathrm{~Hz}$ to $12 \mathrm{~Hz}$, the beta band is $13 \mathrm{~Hz}$ to around 24 $\mathrm{Hz}$, and finally the gamma band is usually described as $25 \mathrm{~Hz}$ and upwards (Niedermeyer \& Silva, 2005). For this study, the alpha-band is of particular interest. Changes in alpha-band frequencies in brain regions have been previously shown to effect cognitive processes. A 1997 study (Klimesch et al.) found alpha-band power changes on the left side of the scalp correlated to different semantic memory, perceptual encoding and attentional processes. They specifically found changes in the power of the upper band (over $10 \mathrm{~Hz}$ ) during memory retrieval tasks. Later Röhm et al. (2001) found similar results, with changes in alpha power occurring in language comprehension tasks. These studies are notable for correlating alpha power change to cognitive processes. It is generally understood today that alpha oscillation serve as some sort of inhibitory mechanisms that suppress stimulus input. In fact it was specifically shown by Cooper et al. (2003) that alpha power increases when a task requires internally directed attention and results in a suppression of sensory input. This suggests specifically that alpha power increases are directly related to internally driven mental operations. It fact when pre-stimulus alpha is high, participants have a harder time detecting stimuli at all (Ergenoglu et al., 2004). Further support for this hypothesis was found by Klimesch et al. (2007), as they found alpha frequency coherence and synchronization to be directly tied to timing control and inhibition of cortical processes, and claimed that alpha desynchronization led to a release of neural inhibition and the increased spreading of activation between brain areas. The finding that increases in alpha power 
is correlated to neural inhibition and lessens the influence of external stimuli on brain activty, seems to be a robust finding and has been replicated in several more recent studies (Händel et al., 2011; Foxe \& Snyder, 2011; Zauner et al., 2014). If this is true, and if adjuncts require more internal processing than arguments, then one would expect lower alpha power in the argument conditions than in the adjunct conditions.

\subsection{Internally and Externally Motivated Stimuli}

Nakao et al. (2012) provide a meta-analysis comparison on decision making experiments when there exists an objective, but less-predictable, answer to a task (things like making decisions on game shows, such as picking the winning brief case for money), and when there is no clear external answer (such as moral decision making, or choosing a preference). They define the situations with clear objective answers to be mostly externally motivated, meaning that the answer to the task exists outside of the subjective experience of the person making the decision. In contrast, tasks without a clear answer are defined as internally motivated, meaning that their answers are mostly subjective to the person making the decision. This discussion relates to a common question in neuroscience, which is how the human brain makes decisions. Traditionally studies on decision making have largely been discussed in terms of prediction, such as how low predictability leads to uncertainty in answering (Platt \& Huettle, 2008). These tasks usually have outcomes that can be evaluated in terms of correctness on the part of the participant, and focus on probabilistic outcomes or stimuli that are hard to perceive. In these cases though, no matter how difficult the answer might have been to predict or understand on the part of the participant there still exists a correct response to the task. This correct response is external to the participant, 
and the participants try their best to align their prediction with what they believe this correct external response will be.

Nakao et al. (2012) goes on to discuss how these externally guided decision making tasks are interpreted in the reinforcement learning (RL) model. The model describes how the expected value of the answer (defined as the magnitude of the outcome times the probability of that outcome) will bias the person making the decision. This expected value changes based on the prediction error of the task (it takes into account the measure of perceived discrepancies between the expected value and the actual value). This seems intuitive, but the model notably fails to completely account for all brain activity when applied to low predictability tasks as pointed out by Hampton et al. (2008) and Pearson et al. (2011). Noticeably the medial prefrontal cortex (MPFC) activates during the decision tasks with low-probability outcomes as well as during decision making tasks in social situations. These brain areas are not areas that have traditionally been associated with decision making, so their activation is surprising. In fact activity in this area is usually linked to the absence of outside stimulation, and is usually most active in situations like boredom. This seems to contrast with what the RL model predicts, because the model assumes a constant interaction between the person making the decision and the expected values. This activation of the MPFC may be a side-effect of the model not accounting for the large degrees of uncertainty present in these situations, and this uncertainty may force the decision maker to look more inwards than accounted for by the existing model.

Nakao et al. (2012) concludes that these externally guided decisions stand in contrast to internally guided decision making, i.e. when the decision has no objectively correct answer to regulate one's response. Tasks which might be considered internally guided include moral judgements and preference decisions. The RL model cannot describe this kind of decision 
making since there is no predicted external outcome to allow for an expected value. Notably though, when one is faced with such tasks there is a consistent increase in MPFC activation, as well as in the perigenual anterior cingulate cortex (pACC) and posterior cingulate cortex (PCC). Interestingly these are all areas associated with the default-mode network (DMN). Previous research has attributed the DMN as largely responsible for the spontaneous or intrinsic activity of the brain (Nakao et al., 2010). The logical conclusion is that personal bias that occurs in internally guided decision making can largely be attributed to the brain's intrinsic activity, or more simply put the activity that exists when one is at rest. This suggests that resting activity plays a role in dealing with these types of decision making - and that the DMN and the spontaneous activity of the brain play a role in expressing theses personal biases. So an increase in activity in the DMN makes sense with this hypothesis, psychologically based on how internally guided decision are defined which is that the response will mostly be determined by one's own personal biases.

The hypothesis for this project is that it is inherent features of verbs that cause their subsequent arguments to be highly predictable and build an expectation for their appearance in subsequent words, and this will be tested. If it is the case that verbs cue their arguments then they form a prediction that can be judged against an expected value based on what the verb implies, similar to externally guided decision making examples discussed above. So if the stored mental representation of verbs have very high predictive power and the processing of the expected arguments are determined by the verb features, then they should require relatively less personal bias on the part of the person doing the processing, when compared to something that is not clearly predictable like adjuncts. For example, the appearance of the verb's arguments is usually an expectation set by the appearance of the verb itself, and limited by the verb, so any personal 
bias when deciphering them is restricted. In contrast, things that are adjunct phrases that are adjoined to the verb will require more interpretation on the part of the listener, as their role and function is not expected by the verb - they cannot be predicted and measured against the verb in the same way arguments can. The next section will motivate this claim.

\subsection{Treating Argument Structure as Externally Motivated}

Do lexicalized features of verbs, such as argument structure, act similarly to the externally motived stimuli? Indeed the predictive power of lexically specific syntactic information to determine upcoming syntactic structure has been repeatedly shown (Altmann \& Kamide, 1999; Arai \& Keller, 2013; Linzen et al., 2013; Kuperberg \& Jaeger, 2016). So if it is the case that argument structure is lexical, then one would expect to find electrophysiological markers of prediction in EEG recordings. For example, phase-amplitude couplings in the medial frontal cortex have been linked to decision making, between the phase of slow oscillations, such as in the delta and theta bands, with the amplitudes of the faster oscillatory bands, such as alpha and beta - and this particular coupling has been shown to be a mechanism for decision feedback encoding in the brain (Cohen et al., 2009).

The high predictability of lexical verb features suggest that these features may behave like externally guided stimuli. If this is the case, then there should be some indication of this predictability in EEG data. The hypothesis is that these features should demonstrate some indication of a phase-amplitude coupling effect. In addition, based on the findings of Bai et al. (2015), one would expect to find lower alpha power in externally motivated conditions. They found that higher alpha power at the onset of the stimulus lead to an increase in self-relatedness to the stimuli, and conversely a stimulus was perceived as less self-related when alpha power 
was low at the onset of the stimulus. They conclude that high alpha power is associated with selfrelated thinking. This finding should hold true when people interpret words in clauses based on lexical verb features, and since the lexical features are external to the participant's self-motivated activity, a low alpha would be an interesting finding since it could suggest a low degree of selfrelatedness on the part of lexical features.

The additional claim of this project is that information from verb adjuncts are more internally motived than expressed information that is directly required by the verb. It has been suggested that adjuncts, such as non-obligatory prepositional phrases, may rely on strength of association and possibly complex reasoning (Hindle \& Rooth, 1993), in structurally ambiguous clauses.

(a) I saw the man with the telescope.

Sentence (5a) is an example of structural ambiguity. It can mean either the subject saw a man using a telescope, or that he saw a man holding a telescope. This ambiguity is really only solved in the real world with extra-grammatical context, since it is impossible to determine what is intended just from the linguistic string. Studies have also shown that linguistic ambiguity resolution is context sensitive (Tanenhaus et al., 1995) and this resolution can vary based on individual differences (Pearlmutter \& MacDonald, 1995). For example, in the study by Pearlmutter \& MacDonald (1995) participants had relatively similar reading times for sentence (6a), where there was an expected argument (the lawyer). When the argument was replaced with the adjunct by the lawyer, reading span varied based on the individual. 
(a) The witness examined the lawyer

(b) The witness examined by the lawyer was useless.

If it is true that context sensitivity and individual differences are important components of adjunct processing, and that these things play a much smaller role in argument processing, then it stands to reasons that adjunct information has a much lower predictability, when compared to arguments. This could be because there is typically nothing about the verb that could indicate an adjunct will follow, whereas the verb provides cues for upcoming arguments. This low predictability coupled with the context sensitive nature of adjunct information means that adjuncts may behave similarly to internally motivated stimuli. This indicates that adjuncts may be more susceptible to resting state effects than arguments, and would conceivably have stronger alpha power relative to a baseline than arguments.

\subsection{Using Grooming Verbs as Effective Stimuli}

Grooming verbs in English are a type of verb that can alternate between an intransitive frame and transitive frame. This group includes verbs such as wash, dress, and shave. These verbs have been identified specifically as grooming verbs by many sources over the last half century (Levin 1993, Kemmer 1993), because they all deal with grooming and share the same alternation. They are also all considered to have reflexive properties that are inherent in their meaning (Reinhart \& Reuland 1993). These verbs are useful stimuli because of their intransitive/transitive alternation. These verbs have an optionally expressed object and thus alternate between an intransitive and a transitive form. Consider the following statements:

(a) John washed. 
(b) John washed himself.

Example (7a) is often assumed to be supressing the internal argument present in (7b), that is to say that (7a) typically has an understood reflexive meaning (Stojanovic 2003, Konig \& Gast 2002, Reinhart \& Reuland 1993). Both (7a) and (7b) are interpreted reflexively, but grooming verbs do not have to take reflexive internal arguments and can take other regular noun phrases, like in (8a). Grooming verbs can therefore occur in the following two frames and still be completely grammatical:

(a) John washed Michael.

(b) John washed quickly.

This is useful because it means we can form something close to a minimal pair where the two first two words are unchanged but the final words differ in terms of structure. Here quickly is an adjunct of the verb washed and is attached sister to the verb phrase.

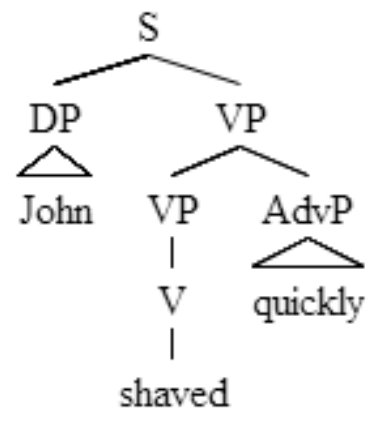

In contrast Michael is clearly an argument of the verb and therefore internal in its structure. 


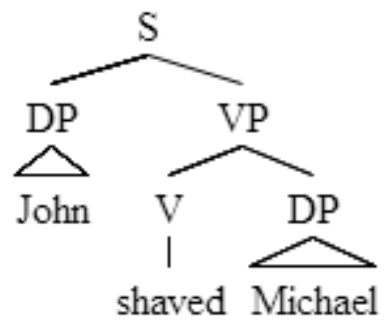

This gives us two conditions which we can use to test whether this difference in structure can be captured on a neuronal level, and whether the difference in neural activation can teach us something about the nature of argument-hood in regards to a rest-stimulus interaction. 


\section{Chapter 4: Methods}

\subsection{Overview and Design}

\subsubsection{Participants}

Twelve volunteers (10 females) participated in the study for monetary compensation ( $\$ 40$ CAD). All participants were healthy individuals with normal vision and were right handed. Ages varied from early to late twenties, with a mean age of 24 . All participants gave their written informed consent to participate in the study. This study was cleared by the Carleton University Research Ethics Board, and all clearance information can be found in the appendix at the end.

\subsubsection{Experimental Design}

There were a total of 16 distinct sentences used as stimuli, and each sentence was repeated during the experiment 15 times. The sentences were all the same number of syllables and roughly the same length. Each sentence employed one of four grooming verbs, that either occurred with a direct object or an adverb, or nothing. The verbs were all controlled for frequency using the Corpus of Contemporary American English. Half of the conditions were semantically odd (coded below as "Non-sense") in meaning and half were well-formed. Example stimuli include:

Table 1

Example of each condition defined by category and type.

\begin{tabular}{llll}
\hline Condition & Category & Type & Code \\
\hline Today in the morning John washed tightly. & Non-sense & Adjunct & NonADJt \\
Today in the morning John washed pencils. & Non-sense & Argument & NonARGt \\
Today in the morning John washed quickly. & Well Formed & Adjunct & nrvADJt \\
Today in the morning John washed Michael. & Well Formed & Argument & nrvARGt \\
& & &
\end{tabular}


Table 1 provides examples of all the target conditions, their category, type, and the code used to label the condition on all figures. A full list of stimuli can be found in the appendix. The stimuli were digitally recorded by a native English female speaker at a normal speaking rate, using Praat (Boersma \& Weenink, 2015).

\subsection{Procedure}

Participants were seated facing a screen at a distance of about $50 \mathrm{~cm}$ and listened with ear buds. The stimuli were divided into four blocks. Each block had eight different sentences, four target conditions and four distractor sentences. Every sentence was repeated 15 times in a random order in each block, for a total of 120 sentences heard in a block and total of 480 heard in the entire session. After hearing each sentence participants were asked to hit a button to indicate they had understood the sentence. Immediately after hitting the button saying they understood, they were asked whether the subject of the sentence was affected in any way. Specifically they were asked whether John was affected in the previous sentence and they answered yes or no. This was done to keep them focused on the task and check that they were paying attention.

\subsubsection{Electrophysiological recording}

EEG data was recorded using a Neuroscan amplifier (Compumedics Neuroscan, Charlotte, $\mathrm{NC}, \mathrm{USA}$ ) and $\mathrm{Ag} / \mathrm{AgCl}$ electrodes through a 64-channel cap (according to the International Ten-Twenty System) referenced to the tip of the nose of the participants. The data was sampled at $1000 \mathrm{~Hz}$ with DC recording. The impedance of each electrode was kept under 5 $\mathrm{K} \Omega$ and $60 \mathrm{~Hz}$ notched. An electrooculogram was recorded for each participant with a pair of 
electors above and below the right eye, and another pair on the outside of each eye. The EEG data pre-processing was performed using the EEGLAB toolbox for MATLAB. The CB1 and CB2 channels were deleted from the data because of irrelevance, and then the data was referenced to two mastoid channels (M1 and M2). The data was filtered with a low-pass filter at $60 \mathrm{~Hz}$ and a high-pass filter at $1 \mathrm{~Hz}$. Epochs were extracted starting $1000 \mathrm{~ms}$ before the onset of the target stimulus and $1000 \mathrm{~ms}$ after the onset. The stimulus onset was locked to the beginning of the final word in each condition.

\subsubsection{Artifact Rejection}

Epochs with unusually high noise were rejected using inference from visual inspection. Other artifacts such as eye blinks and muscle related potentials were left in the data for the independent component analysis (ICA). An extended runica ICA was performed on the data to create 62 ICs time locked to the condition onsets. A visual inspection of IC determined which components were the result of electrode and physiological noise. Rejection was based on multitrial event-related potential (ERP) image-plots, and time course data. These artifacts were rejected from each dataset collected from each participant.

\subsubsection{Event Related Potential Analyses}

To obtain ERP data, data was precomputed with baseline correction from $-1000 \mathrm{~ms}$ to 0 ms relative to the onset of the relevant stimuli, then filtered at $10 \mathrm{~Hz}$ low pass. The data was then averaged across participants and across trials. The built-in EEGLAB statistic software was used to carry out paired t-tests with a permutation for the statistical analysis to compare the two nonsense conditions, with a threshold of $p<.05$ for significance.

\subsubsection{Spectral Analyses}


The spectral analysis is a representation of the overall power of the different frequencies for a given condition. This was calculated using the built-in spectra precomputation of the EEGLAB toolbox. The built-in EEGLAB statistic software was used to carry out a one-way ANOVA with a permutation for the statistical analysis to compare all four conditions, with a threshold of $p<.05$ for significance.

\subsubsection{Event Related Spectral Perturbation Analyses}

ERSP data is a representation of changes in spectral power relative to common baseline in terms of change of time and frequency. This was calculated using the built-in ERSP precomputation of the EEGLAB toolbox. The EEGLAB toolbox uses the mathematical details given by Delorme and Makeig (2004) for ERSP calculation. Again the built-in EEGLAB statistic software was used to carry out a one-way ANOVA with a permutation for the statistical analysis to compare all four conditions, with a threshold of $p<.05$ for significance.

\subsubsection{Inter Trial Coherence Analyses}

The ITC data is a representation of the strength of the coherence of the frequency values across trials. This was calculated using the built-in ITC precomputation of the EEGLAB toolbox. The EEGLAB toolbox uses the same the wavelet mathematical details for ERSP calculation, as the ITC calculation. Again the built-in EEGLAB statistic software was used to perform a paired $\mathrm{t}$-test with a permutation for the statistical analysis to compare the combined argument conditions against the combined adjunct conditions, with a threshold of $p<.05$ for significance. 


\section{Chapter 5: Results}

\subsection{Behavioural results}

The reaction time of the four target conditions were analysed using both descriptive sand inferential statistics.

\subsubsection{Descriptive statistics}

Table 2 provides the mean of each of the conditions and the standard derivation.

Table 2

Means and Standard Deviations of the reaction time for each condition.

\begin{tabular}{lcc}
\hline \multicolumn{1}{c}{ Sentence Type } & \multicolumn{2}{c}{ Reaction Time (ms) } \\
\cline { 2 - 3 } & $\mathrm{M}$ & $\mathrm{SD}$ \\
\hline Well-formed Argument & 273 & 298 \\
Non-sense Argument & 205 & 299 \\
Well-formed Adjunct & 390 & 425 \\
Non-sense Adjunct & 269 & 361 \\
\hline
\end{tabular}

A visual inspection of Table 2 reveals that the mean is lowest for the non-sense argument condition, which is the sentence with a semantic violation in its direct object and it is highest in the well-formed adjunct condition, which is with a semantically plausible adverb. Also the standard deviation is similar for the argument conditions, and it is highest in well-formed adjunct condition, but higher overall for both adjunct conditions. It should be noted that the large standard deviations were not caused by negative reactions times but are an effect of the fact that there were many short reaction times, and a few long ones extending up to a full second. 


\subsubsection{Effect of Sentence Types}

Since the experiment used a repeated measures design, a Repeated Measures ANOVA was run to check the effect of sentence types (Field 2012). It found a significant effect of condition type, $F(2.11,25.27)=6.55, p<.00$, with a moderate effect size, $\eta^{2}=.35$. Based on the reported statistics, the null hypothesis can be rejected, and it can be concluded that semantic and argument manipulations have an effect on reaction time. However, since the standard deviation is consistently higher than the mean in each condition, there cannot be a meaningful pairwise comparison of the conditions.

\subsection{Electroencephalogram Results}

The results of the electroencephalogram (EEG) recordings were analysed to produce several event related potential (ERP), power spectral, and time-frequency graphs. ERPs represent changes in voltage across time, locked to the onset of a stimulus. The power spectral graph represents the absolute change in wave amplitude across frequencies, and the time-frequency graphs represent different frequency effects across time.

\subsubsection{Event Related Potential Results}

Figure 1 provides the scalp array of ERPs comparing each of the non-sense conditions; a significant value is indicated with grey shading. Each channel is labeled in the top left corner. 


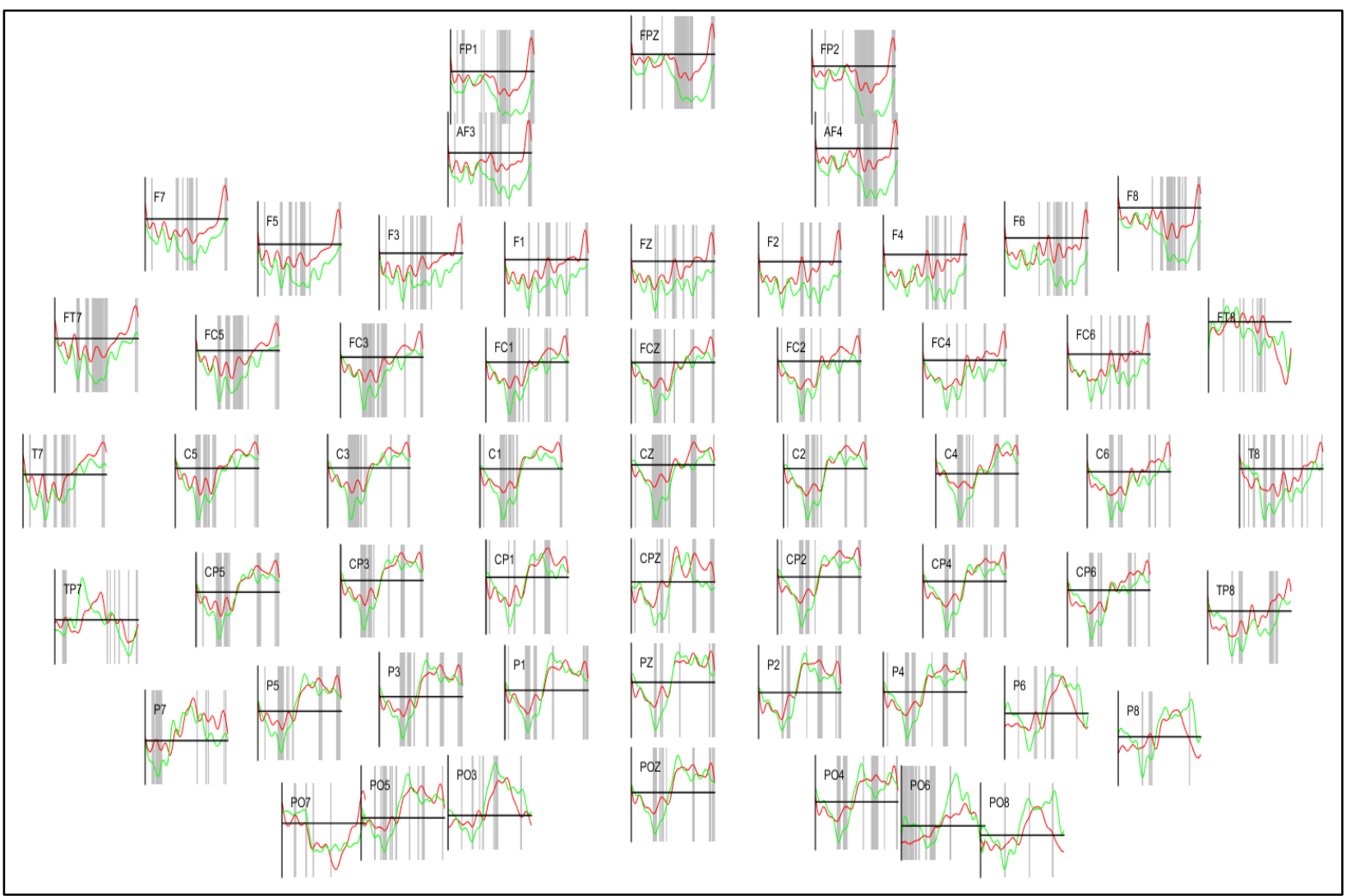

Figure 1. Scalp Array of ERPs.

The scalp array shows that constantly through the scalp there are several significant effects down the middle line and the left frontal regions. Down the middle line there are significant N400 deflections in the non-sense argument condition, indicated by the green lines, and P600 deflections in the non-sense adjunct condition indicated by the red lines. Although there are hints of these deflections throughout the array they seem to be best defined down the middle-line. There also seems to be a clear early left anterior negativity (ELAN) in the left hemisphere in several channels. Most of these ELAN deflections have a significant threshold. A closer inspection on some representative channels below will show a clearer picture.

Figure 2 is the ERP of the $\mathrm{CZ}$ channel. It appears in order to represent the general effects of the midline potentials. Please refer back to Table 1, on page 24, for a list of the condition codes. 

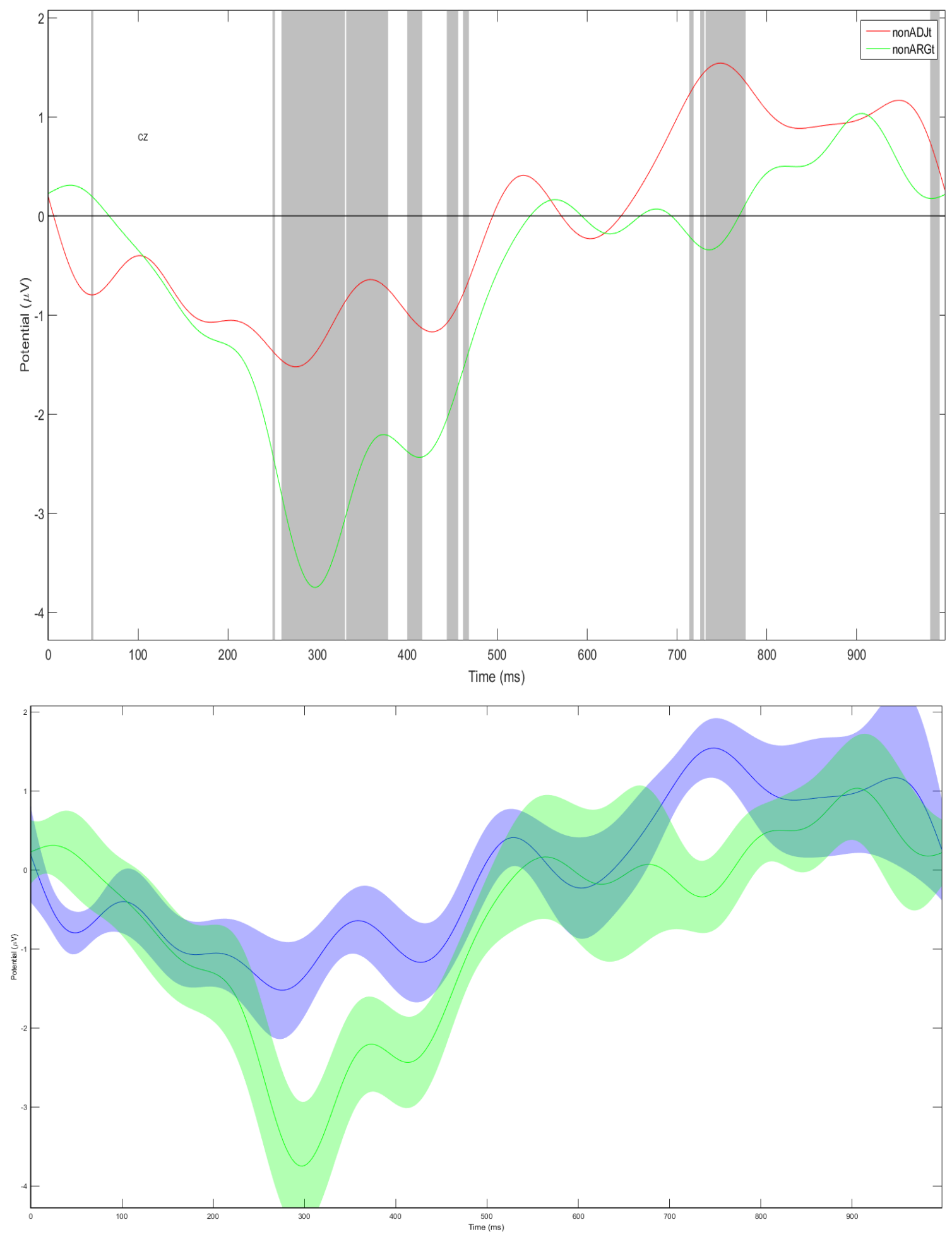

Figure 2. P600 and N400 ERP from channel CZ with degrees of error. 
There seems to be a significant N400 potential originating at about $300 \mathrm{~ms}$ which continues until about $500 \mathrm{~ms}$ for the non-sense argument condition, labeled on the graph as NonARGt. In addition there is a clear P600 potential for the non-sense adjunct condition starting just after 600 $\mathrm{ms}$ and culminating into a significant effect around $750 \mathrm{~ms}$.

The final ERP graph is Figure 3. This graph is displayed as representative of the ELAN component present in the scalp array. 

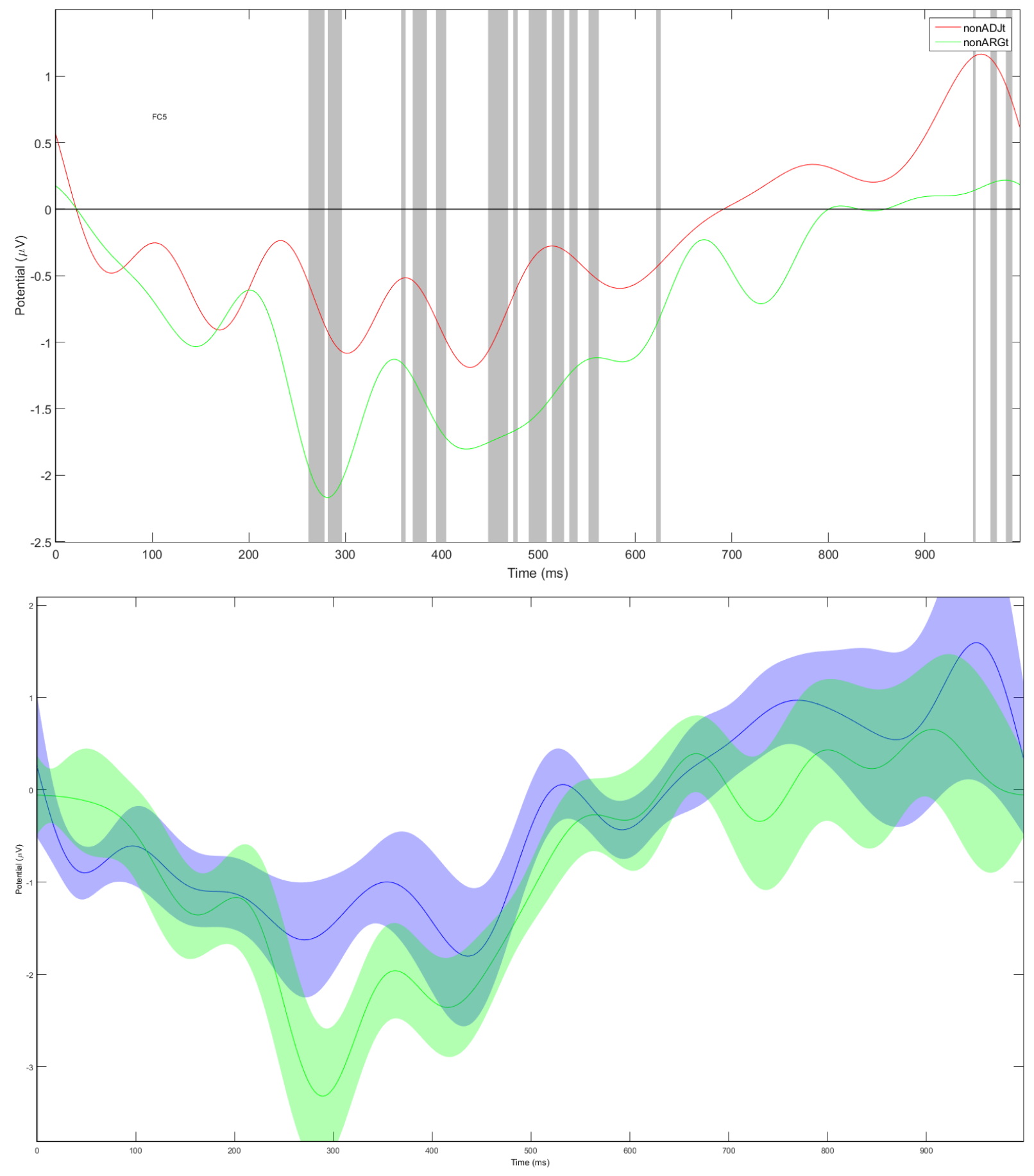

Figure 3. ELAN ERP from channel FC5, and degrees of error. 
Figure 3 seems to clearly show an ELAN originating just after $200 \mathrm{~ms}$, which culminates into a significant effect just before the 300 ms mark. This figure also has hints of significant N400 and P600 responses, but they are not as consistently significant or as visually striking.

\subsubsection{Power Spectra Results}

There are also power spectra results to report, represented in figure 4, taken from the FCZ channel.

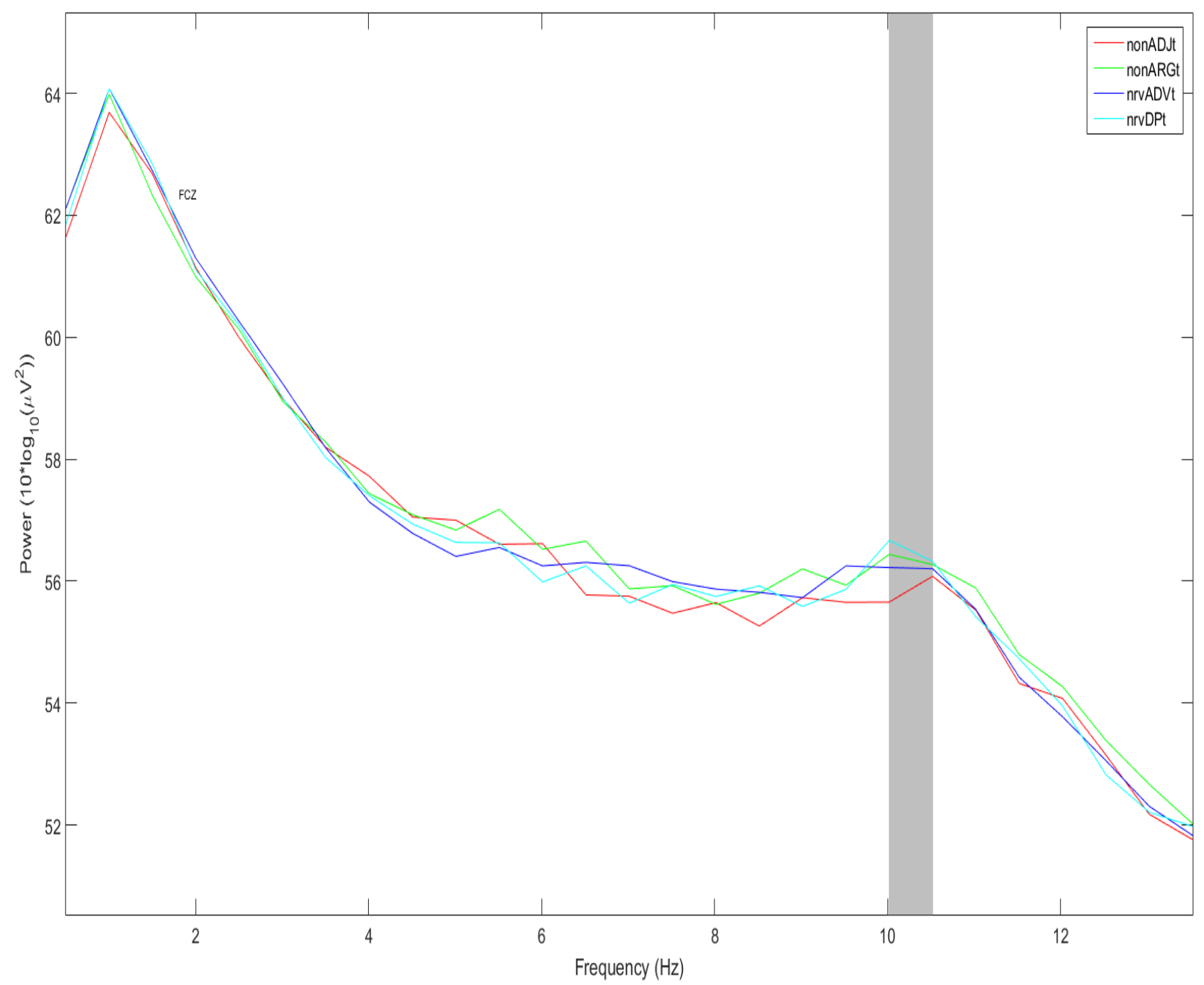

Figure 4. Power Spectral distribution from channel FCZ. 
These results show a significant changes in alpha power in both argument conditions when compared to the adjunct conditions, as well as a significant changes in alpha for the well-formed conditions when compared to their respective non-sense condition.

\subsubsection{Time-Frequency Results.}

The results of the ERSP analysis is presented in figure 5, and like the power spectra results are also taken from the FCZ channel.
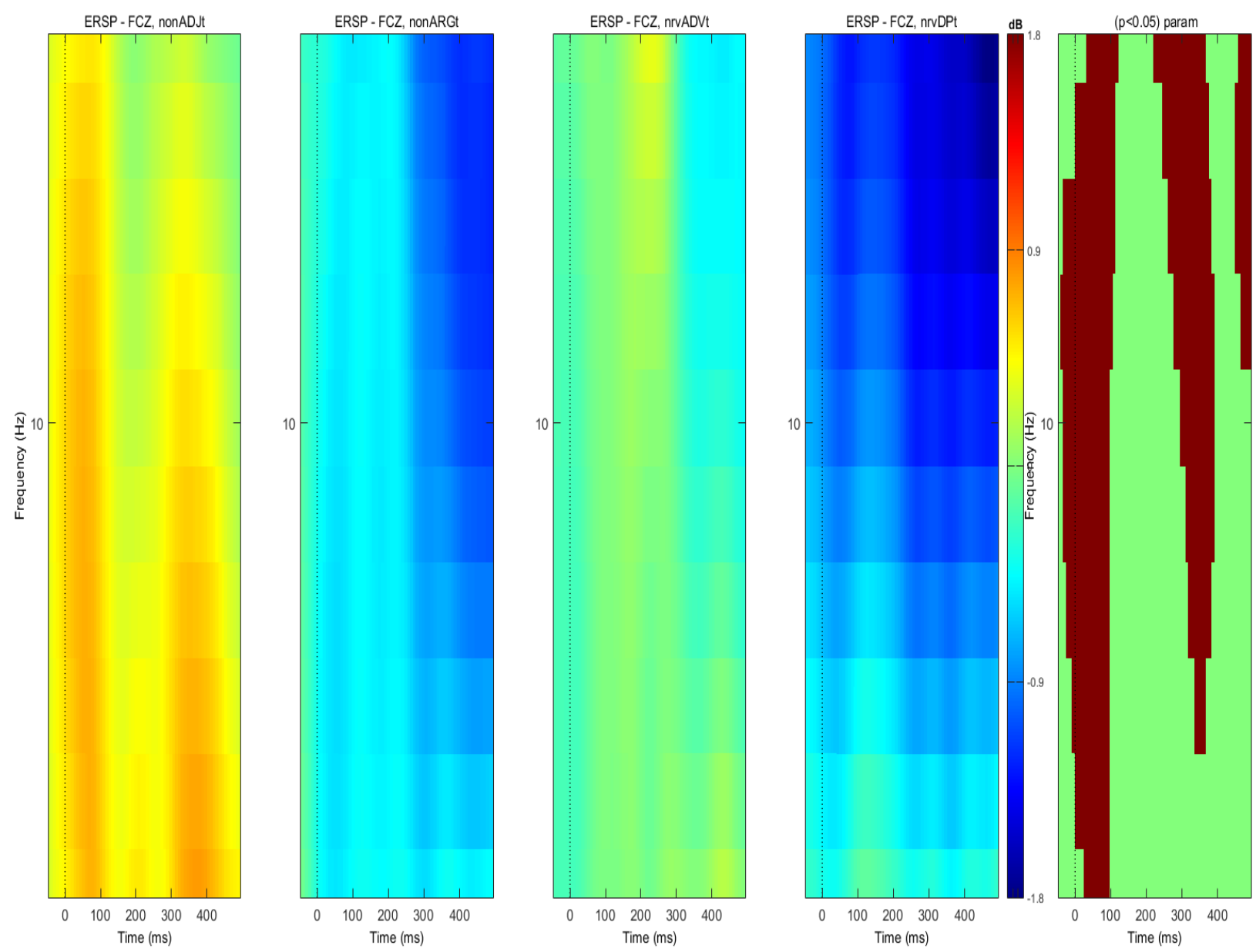

Figure 5. ERSP from channel FCZ.

This figure shows a significant difference between all four conditions in the alpha band, the argument conditions both decrease in alpha after the onset of the stimuli, when compared to the adjunct conditions. Within the argument conditions it seems that the well-formed argument condition has a stronger post-stimulus onset alpha. 
Figure 6 shows the ITC of the two conditions (argument versus adjunct conditions) with the non-sense and the well-formed conditions grouped together in order to have enough trials to generate ITC graphs.
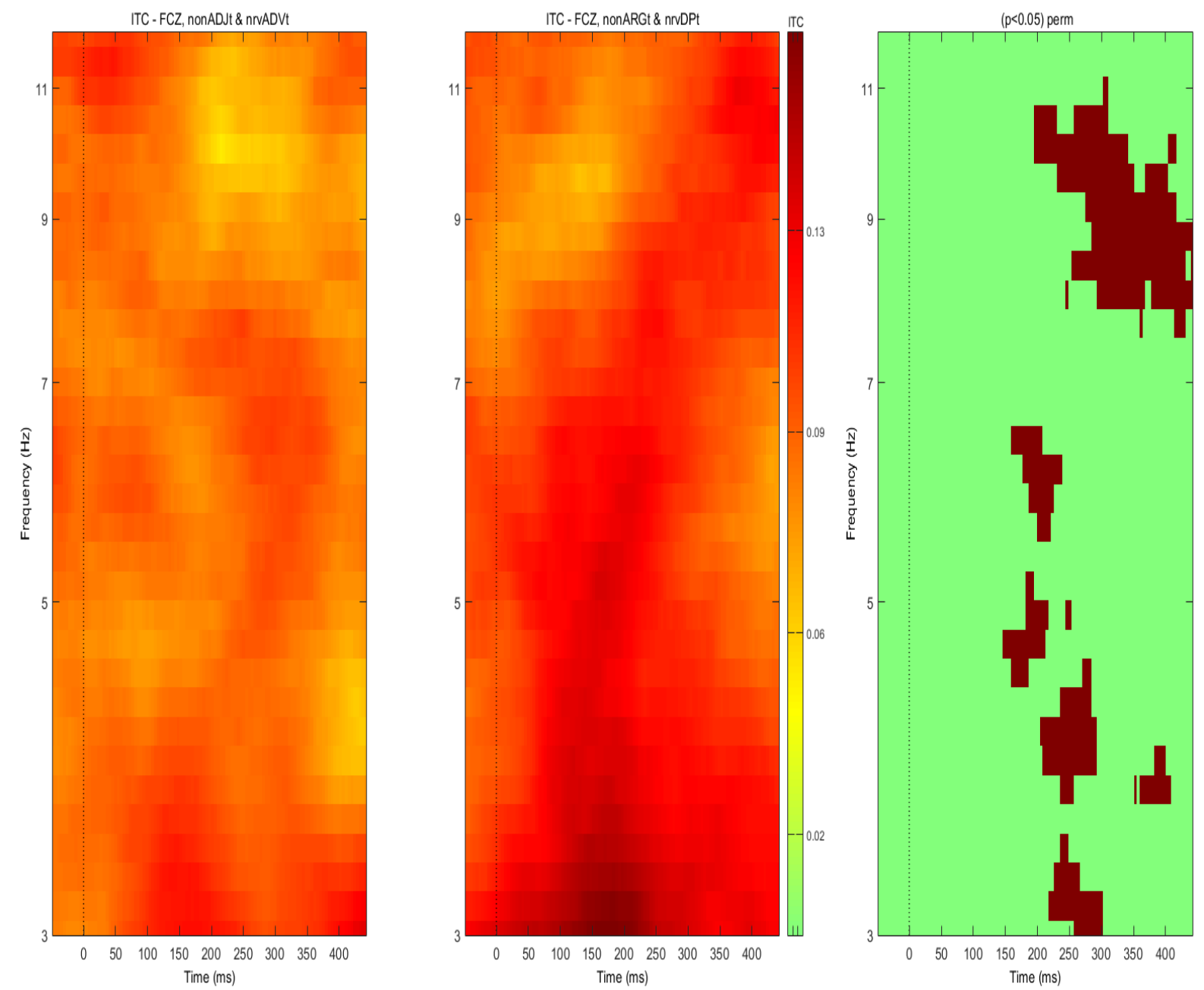

Figure 6. ITC from channel FCZ.

This figure clearly shows a higher amount of inter-trial coherence in the argument condition, with significant values in the delta, theta, and alpha bands. This is an indication of strong temporal structure in the argument condition. 


\section{Chapter 6: Discussion}

\subsection{Overview}

This project is a first attempt to look for the presence of neural prediction mechanisms that shed light on differences in syntactic argument and adjunct processing. The motivating assumption is that online linguistic prediction is a well-documented phenomenon and that this prediction can correlate with other neuronal prediction mechanisms and decision making. The goal was to show verb argument structure as an externally motivated decision making process when predicting subsequent words, and to show this with neuronal evidence, specifically with power changes in the alpha-band. If verb structure causes externally motivated decision-making when predicting upcoming arguments, then the hypothesis was that adjunct information should be unpredictable since its appearance is not determined by verb structure and therefore can only be understood in the linguistic and extra-linguistic context of the whole phrase. The hypothesis is that adjuncts require extra processing, when compared to arguments, because of their unpredictability and unexpectedness. This extra processing is due to a lack of an external cue from the verb to form a prediction before the arguments appear. The lack of an external cue may force a more internally driven processing, and therefore should trigger neural correlates of internal processing, such as high alpha power in response to the adjuncts.

The current study yielded several different results. First there were behavioural differences between the argument and adjunct conditions in reaction time. Secondly, there were also ERP differences between the non-sense argument and adjunct conditions that suggest the nature of the semantic violations in the two conditions are distinct. The analysis continued with an investigation into frequency based effects. There were several significant effects found in the alpha band. There were clear differences in spectra power between conditions, with significant 
change in alpha power in both the argument conditions. The ERSP analysis showed different time-frequency trends in the alpha band in the argument conditions compared to the adjunct conditions. Specifically there was a lowering of alpha power after the stimulus onset in the argument condition. The final analysis was inter-trial coherence (ITC) graph, with the two argument conditions collapsed into a single condition, with the two adjunct ones also collapsed, in order to feed the ITC analysis enough data to accurately to compute accurately. All of these analyses and their findings are described in more detail in the subsequent sections.

\subsection{Interpretation of Behavioural Data}

As shown in table 1, there was a significant effect of the conditions on reaction time, and perhaps more interestingly there were large differences in standard deviation between the argument-type conditions and the adjunct-type conditions. A visual inspection of table 1 shows very similar standard deviations in the two argument conditions, implying that the amount of variability caused by the argument conditions is similar. The standard deviation is also higher in the both adjunct conditions when compared to both argument conditions. It is also worth noting that the standard deviation of the well-formed adjunct condition is also substantially higher than the non-sense adjunct condition. This makes sense with the initial hypothesis that adjuncts have little to no predictability, since the lack of determination from the verb means individuals will have to integrate the adjunct themselves. The presented finding is also in line with the previously discussed work that showed that individual differences play a role in interpreting adjunct information. It is also telling that regardless of the semantic content of the argument presented, the degree of variability largely does not change. The behavioural results therefore support the idea that individual differences have a lesser impact in integrating the argument conditions. The 
explanation for this effect is that the verb creates strong predictions for subsequent words, which lowers the effect of the individual. In summary the difference in predictability between the argument and adjunct conditions is demonstrated by the differences in standard deviations between them. This particular finding would be interesting to replicate in a larger study with a larger sample size with a focus on collecting behavioural data. For now though, I conclude that the behavioural evidence shows a difference between the argument conditions on the one hand and the adjunct conditions on the other.

\subsection{Interpretation of Event Related Potential Data}

The ERP data compared the two non-sense conditions hypothesizing that they would elicit distinct voltage potentials, despite the appearance of a semantic violation in both conditions at the same point in the string. Since both conditions contain a semantic violation, a distinct difference in potentials during its processing would suggest that they are processed differently and that the conditions are distinct. Figure 2 shows differences in event potentials between the conditions. The presence of a N400 deflection in the argument condition relative to the adjunct condition suggests a difference in the semantic integration of the violation between conditions (Friederici \& Frisch , 2000). This could only be the case if the argument is processed differently from the adjunct. The argument condition also elicits an ELAN, as presented in figure 3. This is again in contrast to the adjunct condition which has no such deflection. As previously discussed an ELAN deflection is believed to signify a difference in early syntactic processing, reinforcing the idea that these two conditions are different. Moreover, there is also a P600 in the adjunct condition argument condition. Previous literature claims that P600 effects signify some sort of difference in late syntactic processing, or reanalysis, between the conditions (Friederici \& Frisch, 
2000). This is all to say that the ERP data strongly supports the hypothesis that these two conditions are processed differently. The ERP data in isolation does not provide clear insight on how the conditions are different, but it is strong evidence that they are different.

\subsection{Interpretation of the Power Spectra Data}

Quantitative measures can shed some light on the reasons behind the differences in processing between the argument and adjunct. The results show that the argument conditions have more change in spectral power in the high alpha-band (10-12 Hz) than the adjunct conditions. The data also shows that well-formed conditions have more alpha-band power change than their respective non-sense conditions. Power in this context refers to the absolute change in amplitude of a particular frequency - it is an indication of how active that frequency is. As discussed in an earlier section, high change in power in the high alpha range has been associated with general semantic retrieval, so this finding is not surprising. Beyond just change in power, increases in alpha power, as previously noted, have also been consistently associated with the neuron inhibition and internally motivated tasks, and as noted decreases in alpha power seem to correlate to with more externally motived tasks. The spectra graph however does not provide the insight in how the power changed relative to the stimuli, it is just an overall representation of change; it is displaying the magnitude of the absolute value of the amplitude in that band. In order to understand how the power is shaped by the stimuli an analysis of the power relative to a baseline is necessary and the event-related spectral perturbation analysis fills this role. 


\subsection{Interpretation of Time-Frequency Data}

The event-related spectral perturbation (ERSP) graph in figure 5 provides insight into the relative changes in alpha power before and after the stimuli. The figure shows a strong decrease in alpha power after the onset of the stimuli in the argument condition and a moderate increase in alpha in the adjunct conditions. This finding is in line with the initial prediction of arguments being more externally motivated, as a decrease in the alpha power is associated with stimulus induced activity. An increase in alpha though, like presented in figure 5, is associated with neural inhibition and a reduction of stimulus influence. As has been mentioned this also correlates with self-relatedness and inward thought. This finding further strengthens the hypothesis that adjuncts rely more on individual interpretation to resolve their meaning in the context of the phrase in which they appear.

The inter-trial coherence data is also very interesting. An examination of figure 6 shows that there is much stronger special-temporal structural coherence in the argument condition than the adjunct condition. This suggests less individual variance in the processing of the argument condition. Further investigation may reveal that this is the result of the neural activity aligning itself closely to the stimuli providing less room individual differences to come into effect. The adjunct condition has much less coherence and again this implies a higher degree of variance between the participants, and once again suggesting that the brain is responding less directly to the stimulus and instead is influenced heavily by its spontaneous activity. 


\section{Chapter 7: Conclusion}

The presented evidence indicates that there are different neural mechanisms for argument processing than adjunct processing, and this difference is closely tied to differences in their predictability. Arguments are processed as highly expected information predicted from the lexical specifications of the verb. When arguments are processed there is a strong dip in alpha activity, which suggests a highly stimulus driven process with little self-related input. This agrees with what we know about arguments linguistically speaking, that their appearance is grammatically motivated and they are interpreted in the context of the verb. This is in contrast to adjuncts, which are interpreted in relation to the entire verb phrase, not just the verb - and their appearance is not required and only contextually motivated. This is reflected in the neural activity generated in their processing. Adjuncts seem to have a slight increase in alpha-band power after their onset. This suggests more inward thinking and self-relatedness in their processing.

There are several problems with the current study. Most significantly, there were relatively few trials for the ERSP and ITC analyses. Also the low amount of trials limited other quantitate analyses that could have been performed. Twelve participants is also very low for behavioural analyses, and more participants would improve the statistical power of the reaction time data. A follow up study should include at least double the amount of participants if it were to have a behavioural component, and at least 120 trials per condition. With more trials a new study could build on these findings and try to associate specific mechanism to these two processing types, such as coupling effects across frequency bands, phase locking and resetting, and even the effect of the frequency phase at the onset of the stimuli on interpreting self- 
relatedness. This project is a promising start, but there is room for improvement and much further work would that could be done. 


\section{References}

Altmann, G. T., \& Kamide, Y. (1999). Incremental interpretation at verbs: Restricting the domain of subsequent reference. Cognition, 73(3), 247-264.

Arai, M., \& Keller, F. (2013). The use of verb-specific information for prediction in sentence processing. Language and Cognitive Processes, 28(4), 525-560.

Bai, Y., Nakao, T., Xu, J., Qin, P., Chaves, P., Heinzel, A., \& Northoff, G. (2015). Resting state glutamate predicts elevated pre-stimulus alpha during self-relatedness: A combined EEG-MRS study on "rest-self overlap". Social Neuroscience, 1-15.

Barbu, R. M. (2015). Verbs and Participants: Nonlinguists' Intuitions (Master's thesis, Carleton University Ottawa).

Boeckx, Cedric \& Kleanthes K. Grohmann. 2007. The Biolinguistics manifesto. Biolinguistics 1, 1-8.

Boland, J. E. (2005). Cognitive mechanisms and syntactic theory: Arguments against adjuncts in the lexicon. In Cutler, A. E. (ed.), Psycholinguistic Interfaces. Erlbaum, UK: Taylor and Francis.

Boland, J. E., \& Blodgett, A. (2006). Argument status and PP-attachment. Journal of Psycholinguistic Research, 35(5), 385-403.

Britt, M. A. (1994). The interaction of referential ambiguity and argument structure in the parsing of prepositional phrases. Journal of Memory and Language, 33(2), 251-283.

Paul Boersma \& David Weenink (2013): Praat: doing phonetics by computer [Computer program]. Version 6.0.04, retrieved 1 November 2015 from http://www.praat.org/ Canolty, R. T., \& Knight, R. T. (2010). The functional role of cross-frequency coupling. Trends in Cognitive Sciences, 14(11), 506-515. 
Chomsky, N. (1959). A review of BF Skinner's Verbal Behavior. Language, 35(1), 26-58.

Chomsky, N. (1995). The minimalist program (Vol. 28). Cambridge, MA: MIT press.

Chomsky, N. (2000). New horizons in the study of language and mind. Cambridge: Cambridge University Press.

Chomsky, N. (2005). Three factors in language design. Linguistic Inquiry, 36(1), 1-22.

Cohen, M. X., Elger, C. E., \& Fell, J. (2009). Oscillatory activity and phase-amplitude coupling in the human medial frontal cortex during decision making. Journal of Cognitive Neuroscience, 21(2), 390-402.

Cooper, N. R., Croft, R. J., Dominey, S. J., Burgess, A. P., \& Gruzelier, J. H. (2003). Paradox lost? Exploring the role of alpha oscillations during externally vs. internally directed attention and the implications for idling and inhibition hypotheses. International Journal of Psychophysiology, 47(1), 65-74.

Delorme, A., \& Makeig, S. (2004). EEGLAB: an open source toolbox for analysis of single-trial EEG dynamics including independent component analysis. Journal of Neuroscience Methods, 134(1), 9-21.

den Ouden, D. B., Fix, S., Parrish, T. B., \& Thompson, C. K. (2009). Argument structure effects in action verb naming in static and dynamic conditions. Journal of Neurolinguistics, 22(2), 196-215.

Di Sciullo, A. M., \& Boeckx, C. (Eds.). (2011). The biolinguistic enterprise: New perspectives on the evolution and nature of the human language faculty. Oxford: Oxford University Press. 
Ergenoglu, T., Demiralp, T., Bayraktaroglu, Z., Ergen, M., Beydagi, H., \& Uresin, Y. (2004). Alpha rhythm of the EEG modulates visual detection performance in humans. Cognitive Brain Research, 20(3), 376-383.

Fedorenko, E., \& Kanwisher, N. (2009). Neuroimaging of language: why hasn't a clearer picture emerged? Language and Linguistics Compass, 3(4), 839-865.

Friederici, Angela D., and Stefan Frisch (2000). "Verb argument structure processing: The role of verb-specific and argument-specific information." Journal of Memory and Language, 43(3), 476-507.

Field, A. (2012). Discovering statistics using IBM SPSS Statistics (4th ed.). London: SAGE Publications.

Forker, D. (2014). A canonical approach to the argument/adjunct distinction. Linguistic Discovery, 12(2), 27-40.

Foxe, J. J., \& Snyder, A. C. (2011). The role of alpha-band brain oscillations as a sensory suppression mechanism during selective attention. Frontiers in Psychology, 2, 154.

Grimshaw, J. (1990). Argument structure, Cambridge: The MIT Press.

Hampton, A. N., Bossaerts, P., \& O'Doherty, J. P. (2008). Neural correlates of mentalizingrelated computations during strategic interactions in humans. Proceedings of the National Academy of Sciences, 105(18), 6741-6746.

Händel, B. F., Haarmeier, T., \& Jensen, O. (2011). Alpha oscillations correlate with the successful inhibition of unattended stimuli. Journal of Cognitive Neuroscience, 23(9), 2494-2502.

Hindle, D., \& Rooth, M. (1993). Structural ambiguity and lexical relations. Computational Linguistics, 19(1), 103-120. 
Kemmer, S. (1993). The middle voice (Vol. 23). Amsterdam: John Benjamins Publishing.

Kinsella, A. R. (2009). Language evolution and syntactic theory (Vol. 1). Cambridge: Cambridge University Press.

Klimesch, W., Doppelmayr, M., Pachinger, T., \& Russegger, H. (1997). Event-related desynchronization in the alpha band and the processing of semantic information. Cognitive Brain Research, 6(2), 83-94.

Klimesch, W., Sauseng, P., \& Hanslmayr, S. (2007). EEG alpha oscillations: the inhibitiontiming hypothesis. Brain Research Reviews, 53(1), 63-88.

König, E., \& Gast, V. (2002). Reflexive pronouns and other uses of self-forms in English. Zeitschrift für Anglistik und Amerikanistik, 50(3), 1-14.

Kroeger, P. (2004). Analyzing syntax: a lexical-functional approach. London, UK: Cambridge University Press.

Kuperberg, G. R., \& Jaeger, T. F. (2016). What do we mean by prediction in language comprehension?, Language, Cognition and Neuroscience, 31(1), 32-59.

Kutas, M., \& Federmeier, K. D. (2011). Thirty years and counting: Finding meaning in the N400 component of the event related brain potential (ERP). Annual Review of Psychology, 62, 621.

Larson, R. K. (1988). On the double object construction. Linguistic inquiry, 19(3), 335-391.

Lee, J., \& Thompson, C. K. (2011). Real-time production of unergative and unaccusative sentences in normal and agrammatic speakers: An eyetracking study. Aphasiology, 25(67), 813-825.

Levin, B. (1993). English verb classes and alternations: A preliminary investigation. Chicago, IL: University of Chicago press. 
Levin, B., \& Hovav, M. R. (2005). Argument realization. London, UK: Cambridge University Press.

Linzen, T., Marantz, A., \& Pylkkanen, L. (2013). Syntactic context effects in visual word recognition: An MEG study. The Mental Lexicon, 8(2), 117-139.

Lorenzo, G. (2003). Minimizing the genes for grammar. The minimalist program as a biological framework for the study of language. Lingua, 113(7), 643-657.

Marantz, A. (1984). On the nature of grammatical relations. Linguistic Inquiry Monographs Cambridge, Mass., (10), 1-339.

Marantz, A. (2005). Generative linguistics within the cognitive neuroscience of language. The Linguistic Review, 22(2-4), 429-445.

Meltzer-Asscher, A., Schuchard, J., den Ouden, D. B., \& Thompson, C. K. (2013). The neural substrates of complex argument structure representations: Processing "alternating transitivity" verbs. Language and Cognitive Processes, 28(8), 1154-1168.

Nakao, T., Bai, Y., Nashiwa, H., \& Northoff, G. (2013). Resting-state EEG power predicts conflict-related brain activity in internally guided but not in externally guided decisionmaking. Neuroimage, 66, 9-21.

Nakao, T., Ohira, H., \& Northoff, G. (2012). Distinction between Externally vs. Internally Guided Decision-Making: Operational Differences, Meta-Analytical Comparisons and Their Theoretical Implications. Frontiers in Neuroscience, 6, 31-31.

Nakao, T., Osumi, T., Ohira, H., Kasuya, Y., Shinoda, J., Yamada, J., \& Northoff, G. (2010). Medial prefrontal cortex-dorsal anterior cingulate cortex connectivity during behavior selection without an objective correct answer. Neuroscience letters, 482(3), 220-224. 
Needham, Stephanie, and Ida Toivonen, (2011). Derived Arguments. In Miriam Butt and Tracy Holloway King, eds., Proceedings of the LFG11 Conference, 401-421. Stanford, CA: CSLI Publications.

Niedermeyer, E., \& da Silva, F. L. (Eds.). (2005). Electroencephalography: basic principles, clinical applications, and related fields. Philadelphia, PN: Lippincott Williams \& Wilkins.

Pearlmutter, N. J., \& MacDonald, M. C. (1995). Individual differences and probabilistic constraints in syntactic ambiguity resolution. Journal of Memory and Language, 34(4), 521-542.

Pearson, J. M., Heilbronner, S. R., Barack, D. L., Hayden, B. Y., \& Platt, M. L. (2011). Posterior cingulate cortex: adapting behavior to a changing world. Trends in cognitive sciences, $15(4), 143-151$.

Phillips, C., \& Sakai, K. L. (2005). Language and the Brain. Yearbook of Science and Technology. New York, NY: McGraw-Hill Publishers.

Platt, M. L., \& Huettel, S. A. (2008). Risky business: the neuroeconomics of decision making under uncertainty. Nature Neuroscience, 11(4), 398-403.

Poeppel, D. (2012). The maps problem and the mapping problem: two challenges for a cognitive neuroscience of speech and language. Cognitive Neuropsychology, 29(1-2), 3455.

Poeppel, D., \& Embick, D. (2005). Defining the relation between linguistics and neuroscience. Twenty-first century psycholinguistics: Four cornerstones, 103-118.

Pollard, C., \& Ivan, A. (1994). Head-driven phrase structure grammar. Stanford: Center for the Study of Language and Information. 
Reinhart, T., \& Reuland, E. (1993). Reflexivity. Linguistic Inquiry, 24(4), 657-720.

Röhm, D., Klimesch, W., Haider, H., \& Doppelmayr, M. (2001). The role of theta and alpha oscillations for language comprehension in the human electroencephalogram. Neuroscience Letters, 310(2), 137-140.

Schütze, C. T., \& Gibson, E. (1999). Argumenthood and English prepositional phrase attachment. Journal of Memory and Language, 40(3), 409-431.

Shapiro, L. P., Zurif, E. B., \& Grimshaw, J. (1989). Verb processing during sentence comprehension: Contextual impenetrability. Journal of Psycholinguistic Research, 18(2), 223-243.

Stojanovic, D. (2003). I shave just like man': intrinsic and extrinsic reflexive constructions in child English. In Proceedings of the 2002 Annual Canadian Linguistic Association Meeting (pp. 312-325).

Sidtis, D. V. L. (2007). Reprint of "Does functional neuroimaging solve the questions of neurolinguistics?"[Brain and Language 98 (2006) 276-290]. Brain and language, $102(2), 200-214$

Tallerman, M. (2014). Understanding syntax. Abingdon: Routledge.

Tanenhaus, M. K., Spivey-Knowlton, M. J., Eberhard, K. M., \& Sedivy, J. C. (1995). Integration of visual and linguistic information in spoken language comprehension. Science, 268(5217), 1632-1634.

Thompson, C. K., Bonakdarpour, B., Fix, S. C., Blumenfeld, H. K., Parrish, T. B., Gitelman, D. R., \& Mesulam, M. M. (2007). Neural correlates of verb argument structure processing. Journal of Cognitive Neuroscience, 19(11), 1753-1767. 
Tutunjian, D., \& Boland, J. E. (2008). Do we need a distinction between arguments and adjuncts? Evidence from psycholinguistic studies of comprehension. Language and Linguistics Compass, 2(4), 631-646.

Zauner, A., Gruber, W., Himmelstoß, N. A., Lechinger, J., \& Klimesch, W. (2014). Lexical access and evoked traveling alpha waves. NeuroImage, 91, 252-261. 


\section{Appendix A: Stimuli}

The follow is all the stimuli used during the study, with target conditions bolded:

$\underline{\text { Block } 1}$

Today in the morning John shaved

Today in the morning John stripped again

Today in the morning John washed Michael

Today in the morning John shaved deeply

Today in the morning John stripped buttons

Today in the morning John bathed himself

Today in the morning John slapped Michael

Today in the morning John poked himself

$\underline{\text { Block } 2}$

Today in the morning John bathed

Today in the morning John shaved poorly

Today in the morning John stripped Michael

Today in the morning John washed tightly

Today in the morning John bathed pistols

Today in the morning John washed himself

Today in the morning John kicked himself

Today in the morning John cut himself
Block 3

Today in the morning John washed

Today in the morning John bathed quickly

Today in the morning John shaved Michael

Today in the morning John bathed fairly

Today in the morning John shaved robots

Today in the morning John stripped himself

Today in the morning John poked Michael

Today in the morning John slapped himself

\section{$\underline{\text { Block } 4}$}

Today in the morning John stripped

Today in the morning John washed briefly

Today in the morning John bathed Michael

Today in the morning John stripped stupidly

Today in the morning John washed pencils

Today in the morning John shaved himself

Today in the morning John cut Michael

Today in the morning John kicked himself 
Appendix B: Ethics clearance form

\section{Garleton \\ NIV 351 T \\ Cenada's Capitel University}

Ethics Clearance Form - New Clearance
Carleton University

Research Bthics Office

Research Ethics Board

511 Tory, 1125 Colonel By Drive

Ottawa, ON K1S 5B6 Canada

Tel: 613-520-2517, ethics@acarleton.ca

This is to certify that the Carleton University Research Ethics Board has examined the application for ethical clearance. The REB found the research project to meet appropriate ethical standards as outlined in the Tri-Council Policy Statement: Ethical Conduct for Research Imvolving Human, 2nd edition, and the Carleton University Policies and Procedures for the Ethical Conduct of Research.

Date of Clearance: January 29, 2016

Researcher: George Northoff (Primary Investigator)

Daniel Digiovanni (Student Research: Master's Student)

Department: Other External OrganizationlOther External Department

University: University of Ottawa Mental Health Research

Research Supervisor (if applicable): Prof. George Northoff

Project Number: 104099

Project Title: Function of the Medial Prefrontal Cortex [Daniel DiGiovanni]

Funder (if applicable): N/A

Clearance Expires: August 31, 2016

All researchers are governed by the following conditions:

Annual Status Report: You are required to submit an Annual Status Report to either renew clearance or close the file. Failure to submit the Annual Status Report will result in the immediate suspension of the project. Funded projects will have accounts suspended until the report is submitted and approved.

Changes to the project: Any changes to the project must be submitted to the Carleton University Research Ethics Board for approval. All changes must be approved prior to the continuance of the research.

Adverse events: Should a participant suffer adversely from their participation in the project you are required to report the matter to the Carleton University Research Ethics Board. You must submit a written record of the event and indicate what steps you have taken to resolve the situation.

Suspension or termination of clearance: Failure to conduct the research in accordance with the principles of the Tri-Council Policy Statement: Ethical Conduct for Research Involving Humans, 2nd edition and the Carleton University Policies and Procedures for the Ethical Conduct of Research may result in the suspension or termination of the research project.

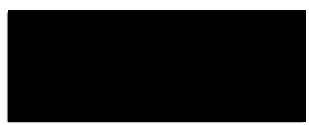

Louise Heslop

Chair, Carleton University Research Ethics Board

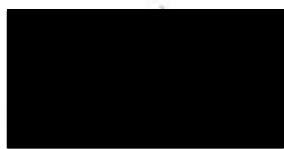

Andy Adler

Vice-Chair, Carleton University Research Ethics Board 